\title{
El autismo en el Perú: Una mirada desde el Equipo de Investigación y Trabajo en Autismo
}

\author{
Autism in Peru: An outlook from autism research and work \\ team
}

Ernesto Reaño Carranza

Equipo de Investigación y Trabajo en Autismo. Lima, Perú

Recibido: 05/01/2022 Revisado: 06/01/2022 Aceptado: 09/01/2022 Publicado: 15/01/2022

\section{RESUMEN}

El Equipo de Investigación y Trabajo en Autismo (EITA) fue fundado en el 2009 por el Mg. Ernesto Reaño con el objetivo de brindar servicios de apoyo a la comunidad autista, cuidadores y familiares y trabajar en pro de la implementación de ajustes y apoyos en los diversos contextos sociales buscando la accesibilidad de esta población. EITA es pionera en el Perú en el trabajo desde el paradigma de la neurodiversidad, la implementación de servicios adecuados para cada persona autista respetando su edad de desarrollo, orientación sexual, identidad de género, identidad cultural, etc. sin ningún tipo de discriminación. En ese sentido, se enmarca dentro del apoyo constante al reconocimiento de los derechos humanos de la persona autista y al movimiento de la neurodiversidad. Palabras clave: autismo, condiciones del espectro autista, neurodiversidad, derechos humanos.

\begin{abstract}
EITA was founded in 2009 by Mg. Ernesto Reaño in order to provide support services to the autistic community, caregivers and family members, and working towards the implementation of adjustments and supports in the various social contexts seeking accessibility for this population. EITA is pioneer in Peru working on the neurodiversity paradigm, the implementation of adequate services for each autistic person, respecting their development age, sexual orientation, gender identity, cultural identity, etc. without any discrimination. In this sense, it is part of the constant support for the recognition of the human rights of the autistic person and the neurodiversity movement. Keywords: autism, autism spectrum conditions, neurodiversity, human rights.
\end{abstract}




\section{INTRODUCCIÓN}

El autismo es una condición del neurodesarrollo donde la mente privilegia un tipo de procesamiento basado en la hiperfocalización en detalles, búsqueda de patrones y profundización en áreas de interés; presentando dificultades en el procesamiento de los elementos de la comunicación y de cognición social típicas (Walker, 2014; Milton, 2012, Reaño, 2020). Según cifras de la Organización Mundial de la Salud (2021), se presenta aproximadamente en el $1 \%$ de la población mundial. En el Perú, según un reporte del Ministerio de Salud en 2019, sólo tenemos detectadas 15 625 personas con esta condición, de los cuales el 90,6 \% son menores de 11 años y el 81 $\%$ son de género masculino. Estas cifras se encuentran muy por debajo de las detecciones que deberíamos encontrar según la prevalencia mundial antes descrita, ignoran a la población adulta autista y presenta un severo sesgo de género cuando la proporción estimada actual entre hombres y mujeres autistas es de 3:1 (Loomes, Hull y Mandy, 2017).

La situación a nivel de detecciones y concientización era aún más precaria cuando EITA es fundada. Si bien su conformación oficial se da en en el año 2009, tiene sus precedentes desde el año 2008 en un departamento de la calle Arica en Miraflores, Lima. $\mathrm{Su}$ fundador, Mg. Ernesto Reaño, psicólogo de formación había terminado los estudios de doctorado en Ciencias del Lenguaje por la Université Sorbonne Nouvelle Paris - III, motivado por el Dr. Luis Jaime Cisneros, regresaba a Lima. Las interrogantes sobre el autismo y la forma de percibir y dar sentido al mundo, observadas en el colegio Santa Magdalena Sofía Barat, dedicado a la atención de niños con notables necesidades de apoyo en el lenguaje, constituyeron los moti- vos iniciales para definir y dirigir las líneas de la investigación y tratamiento en autismo.

En sus inicios, EITA, significaba Equipo de Investigación y Tratamiento en Asperger y Autismo. En el año 2018, luego de conocerse la colaboración de Hans Asperger con las políticas de eugenesia y eutanasia del régimen nazi, el nombre cambió. Fue la oportunidad de reflejar años de aprendizaje en la comprensión del autismo como un espectro, de superar la falaz dicotomía "leve"/"severo", de afianzar los orígenes de EITA basados en el paradigma de la neurodiversidad y no en el modelo médico. Pasó a ser Equipo de Investigación y Trabajo en Autismo (EITA).

En un viaje a Chile en 2008, a través de unos colegas, Reaño conoce al $\mathrm{Mg}$. Theo Peeters, quien sería decisivo para la formación de EITA. Peeters, director del Centro de Formación Opleidingscentrum Autisme en Amberes (Bélgica), encarnaba una mirada que iba un paso más allá de la filosofía TEACCH que impulsara en Europa: la empatía radical con la persona autista (El Tiempo, 2011). Su enseñanza incluía muchas reflexiones de autistas en primera persona, cosa poco habitual para aquel entonces. Aún no era una época en la que las voces autistas tenían la difusión, redes y colectivos que podemos encontrar actualmente. Fue un divulgador de la actividad de Jim Sinclair y la Autistic Network International que fundase, de Therese Joliffe, Sean Barron, Gunilla Gerland, Marc Segar, Clare Sainsbury, aparte de voces ya más conocidas en ese momento como las de Temple Grandin y Donna Williams. Dos impulsos aportó, entonces, la enseñanza y amistad de Peeters a EITA desde sus inicios: la noción del autismo como "condición de vida" y la escucha atenta a las voces en primera persona. 
La presencia de Theo Peeters consolidó la mirada y dirección de EITA desde el Primer Seminario - Taller Internacional sobre Asperger y Autismo (2010), quien por primera vez visitó Lima. EITA volvió a realizar dos congresos en los años 2011 y 2012, también con la participación del Theo Peeters e Hilde De Clercq, especialista en autismo y madre de una persona autista, autora de "El autismo desde dentro. Una guía", libro traducido por EITA y presentado en el 2011. Esta obra reforzó la convicción de EITA sobre la necesidad de entender el autismo como una "cultura" hacia la cual se requiere tender puentes y comprender sus formas de procesamiento y percepción del mundo como partes esenciales y complementarias de la diversidad humana.

Con estos precedentes, el camino al paradigma de la "neurodiversidad" era natural. Este concepto- que había nacido de la mano de Judy Singer en 1998- era desconocido o al menos no era utilizado en nuestro país. La neurodiversidad se refiere a la variabilidad neurocognitiva virtualmente infinita dentro de la población humana de la Tierra. Señala el hecho de que cada ser humano tiene un sistema nervioso único con una combinación única de habilidades y necesidades. La neurodiversidad es un subconjunto de la Biodiversidad, un término que se usa principalmente con el propósito de abogar por la conservación de las especies. (Singer, 2016, 2020). En el 2010, en el artículo "Respetemos la neurodiversidad", se expuso por primera vez en nuestro país, de manera pública, el concepto neurodiversidad, que constituye el paradigma de trabajo de EITA.

Esta comunidad, de personas CEA ("condición" y no "trastorno", como veremos), ha acuñado el término neurodiversidad el cual supone que un desarrollo neurológico atípico (neurodivergente) es una diferencia normal que debe ser reconocida y respetada como cualquier otra variante humana. En este sentido, debemos esforzarnos por comprender que no todos procesamos la información de la misma manera, ni damos el mismo peso a lo social, o que, simplemente, existen diversas maneras de ver el mundo: ni mejores ni peores, distintas. Ser neurodivergente es lo opuesto a ser neurotípico ("neurótico típico", el resto de la población).

EITA nació, entonces, bajo la idea la idea de crear servicios de apoyo para la comunidad autista, sus cuidadores y familiares, así como para la difusión y concientización del autismo desde el paradigma de la neurodiversidad en los diferentes contextos (hogar, escuela, espacios públicos, etc.) donde interactuase la persona autista. Nuestra idea siempre fue crear entornos "amigables" para el autismo bajo la certeza de que una sociedad adecuada para la neurodivergencia, en específico para el autismo, podría ser de mayor provecho al crear una accesibilidad que beneficiaría a todos.

Los primeros servicios brindados en EITA incluyeron, además de la detección e intervención, un taller de habilidades sociales para niños, adolescentes y adultos, actualmente de llamado "habilidades sociales neurotípicas". Se ha concebido, como un espacio donde poder aprender las habilidades típicas que les permitan interactuar y protegerse en y del entorno. Asimismo, como un grupo de referencia y reafirmación de su condición. No es común encontrar un grupo de personas autistas al cual pertenecer. Todos los participantes saben de su condición, se saben autistas. Para el enfoque de EITA, el autismo no es un diagnósti- 
co sino una identidad. Se "es" autista como se "es" peruano, es parte sustancial de la manera como uno concibe el mundo y no puede ser separado del individuo, no es algo que se "tenga", como se "tiene" un accesorio o un trastorno.

En el proceso de investigación y trabajo en EITA, en el año 2017 se creó el primer servicio destinado exclusivamente a la detección, intervención y apoyo a mujeres autistas. Es importante entender que el autismo en la población femenina, ha sido largamente invisibilizado debido a concepciones erradas, al ser considerado como mayoritariamente masculino y por las descripciones que involucran sólo características de este género, como apuntásemos al incio de este artículo (Lai, Baron-Cohen y Buxbaum, 2015; Bargiela, Steward y Mandy, 2016). Ser mujer en una sociedad machista y capacitista supone una doble situación de vulnerabilidad: por ser mujer y por ser autista. EITA elaboró la "Guía de sexualidad para mujeres autistas" de autoría de la Lic.Andrea Mesones y la Lic. Luciana Augusto. Este material es pionero en el país y en América Latina. Se dirige a las adolescentes y adultas autistas con un lenguaje sencillo y directo e ilustrado de una manera precisa y amigable, detallando los cambios fenotípicos y hormonales ligados al desarrollo corporal; el deseo y la orientación sexual; la identidad y expresión de género; la salud sexual y reproductiva y la prevención del abuso.

A partir del año 2019, se iniciaron los estudios e implementación de servicios de apoyo en la diversidad sexual e identidad de género en el autismo, en concordancia con estudios que señalan que hay diferencias notables en la orientación sexual (casi el $70 \%$ de la población autista se enuncia como no heterosexual) así como en la identidad y ex- presión de género en la comunidad autista respecto de la neurotípica (entre 3 y 6 veces más identidades de género divergentes). (George y Strokes, 2017; Warrier et al., 2020).

EITA junto con la Defensoría del Pueblo del Perú, ha participado desde el año 2010, en campañas de sensibilización sobre el autismo como condición, la restricción del uso de pirotécnicos y, en especial, sobre educación inclusiva. Colaboramos, también, con el ministerio de Educación para su "Guía para la Atención Educativa de Niños y Jóvenes con Trastorno del Espectro Autista - TEA", en el año 2013. Producto de estas experiencias en el año 2020 se crea el servicio de Área Educativa, el cual tiene como objetivo acompañar los procesos escolares, de orientación vocacional y de educación superior, de las personas autistas de acuerdo con su perfil individual de aprendizaje y favoreciendo que se otorguen los apoyos y ajustes en el acceso a una educación plena.

El compromiso ético de EITA con la sociedad está presente en todas los proyectos y actividades que realiza, dando prioridad al cumplimiento de las libertades y garantías de la comunidad autista como parte del movimiento de la neurodiversidad, en la búsqueda del respeto de los derechos humanos de toda persona neurodivergente. Los principios que rigen la conducta institucional en la defensa del derecho a la educación y la libertad de expresión, se han puesto de manifiesto al EITA haber enfrentado un proceso judicial (https://ernestoreano.pe/ trener-contra-reano/), resuelto a su favor, en el "Caso Trener contra Reaño" (2014-2020) recogido en un libro en proceso de edición.

La pandemia provocada por el COVID-19 y las restricciones que de ella se 
derivaron, generaron nuevos desafíos en las áreas de comunicación. Considerando las experiencias en diversas formas e intentos de comunicación a distancia y las necesidades de los participantes, se ha constatado, con mayor precision, la complejidad y multimodalidad de la comunicación y del lenguaje en el autismo. Es así como a partir del 2022, se inaugura el departamento de "Comunicación, lenguaje y sistemas de comunicación aumentativa alternativa".

El enfoque de trabajo de EITA parte de una profunda reflexión filosófica y ética -siempre en constante renovación- sobre lo que es el autismo y el trabajo con personas autistas.

El autismo no es una enfermedad ni un trastorno, sino una condición de vida con la cual se nace y que supone un neurodesarrollo atípico (Reaño, 2017). A diferencia del cerebro neurotípico (el de la mayoría poblacional) éste está mucho más preparado para las actividades que requieren una mayor capacidad de sistematización de acuerdo con sus motivaciones profundas (Shore, 2012; Reaño, 2017). Su tipo distinto de comprensión de la interacción social y de establecimiento de la empatía, hacen que encuentren dificultades para comprender el funcionamiento de la sociedad neurotípica. Esta levanta serias barreras para su determinación, autonomía y acceso a la calidad de vida (Milton, 2012).

Así, el enfoque de EITA se encuentra dentro del paradigma de la neurodiversidad, en cuanto se señala que existen diferentes tipos de configuraciones cerebrales (neurotipos) y ninguno es mejor o superior a otro, cada uno presenta talentos y dificultades que deben ser superadas mediante los apoyos correspondientes. Se considera, ante todo, la dignidad de la persona autista en cuanto no se busca "normalizarlos" ni que dejen de serlo, sino que accedan a vivir, de manera plena, a sus proyectos de vida particulares.

Para el equipo, las personas autistas son sujetos con los que se trabaja brindando comprensión y apoyo, y no objetos que puedan ser mirados a través de un "tratamiento" predeterminado.

Se evita el enfoque médico y de la patología. Por ello:

1. No son "pacientes" sino "participantes".

2. No se hacen "diagnósticos" sino "detecciones".

3. No se brindan "terapias" sino "acompañamientos”, «intervenciones», en los que se busca crear lazos de empatía, escucha, comprensión y acción.

Para realizar este trabajo se plantean y utilizan herramientas psicoeducativas que permitan a la persona autista y sus cuidadores:

1. Comprender su condición y el respeto a sus diferencias.

2. Comprender las particularidades de su entorno y las habilidades sociales neurotípicas para poder interactuar con el entorno y aprender a protegerse.

3. Comprender sus procesos de aprendizaje y posibilitar su aprendizaje con satisfacción.

4. Brindar la orientación a las escuelas, centros de estudio y de trabajo y demás entornos para realizar las adecuaciones y apoyos necesarios para el desenvolvimiento pleno de la persona autista.

5. Poder realizar un proyecto de vida propio en base a la autonomía, determinación y acceso a los apoyos pertinentes. 
6. Trabajar por el empoderamiento de la persona autista, reconociendo y garantizando el acceso a sus derechos ciudadanos, considerando y respetando toda forma de diversidad y pluriculturalidad.

Se agradece al equipo actual de EITA: Bach. Andrea Mesones, Bach. Marie Saldaña, Lic. Julissa Vernal, Lic. Beatriz Lázaro, Lic. Lourdes López, Mg. Cloe Moreno, Lic. Gabriela Chambi, Lic. Mario Puga, Lic. Luciana Augusto, Lic. Rosa Victoria Chauca, que son parte de nuestra historia, de nuestra memo- ria, pero también de nuestra imaginación.

\section{CONCLUSIONES}

EITA trabaja por la generación de una convivencia respetuosa entre todas las formas de neurodiversidad y por los derechos humanos de la persona autista.

El trabajo de EITA no se resume en un «método» o «terapia» sino en una comprensión profunda filosófica y ética de la persona autista y los apoyos necesarios para cada cual considerando su individualidad, su dignidad y su desarrollo pleno.

\section{REFERENCIAS BIBLIOGRÁFICAS}

Bargiela, S., Steward, R. y Mandy, W. (2016). The Experiences of Late-diagnosed Women with Autism Spectrum Conditions: An Investigation of the Female Autism Phenotype. J Autism Dev Disord 46, 3281-3294 https://doi.org/10.1007/s10803-016-2872-8

El Tiempo (2011). 'El autismo no es una enfermedad'. https://www.eltiempo.com/archivo/documento/MAM-4560809

George, R. y Stokes, M. (2018), Sexual Orientation in Autism Spectrum Disorder. Autism Research 11: 133-141. https://doi.org/10.1002/aur.1892

Lai, M. C., Baron-Cohen, S. y Buxbaum, J. D. (2015). Understanding autism in the light of sex/ gender. Molecular Autism 6, 24 https://doi.org/10.1186/s13229-015-0021-4

Loomes, R., Hull, L., y Mandy, W. P. L. (2017). What Is the Male-to-Female Ratio in Autism Spectrum Disorder? A Systematic Review and Meta-Analysis. J. Am. Acad. Child Adolesc. Psychiatry. 56(6):466-474. doi: 10.1016/j.jaac.2017.03.013

Mesones, A. y Augusto, L. (2019). Guía de sexualidad para mujeres autistas. Ediciones EITA.

Milton, D. (2012) On the ontological status of autism: the 'double empathy problem. Disability \& Society, 27:6, 883-887, DOI: 10.1080/09687599.2012.710008

Milton, D. (2017). So what exactly is autism? http://capacity-resource.middletownautism.com/ wp-content/uploads/sites/6/2017/03/damian-milton.pdf 
Ministerio de Educación del Perú (2013). Guía para la Atención Educativa de Niños y Jóvenes con Trastorno del Espectro Autista - TEA. http://www.minedu.gob.pe/minedu/archivos/a/002/05-bibliografia-para-ebe/5-guia-para-la-atencion-de-estudiantscon-trastorno-del-espectro-autista.pdf

Ministerio de Salud del Perú (2019). El 81 \% de personas tratadas por autismo en Perú son varones. Nota de Prensa. https:/www.gob.pe/institucion/minsa/noticias/27103-el-81-depersonas-tratadas-por-autismo-en-peru-son-varones

Reaño, E. (2010) Respetemos la neurodiversidad. https://ernestoreano.pe/respetemos-la-neurodiversidad/

Reaño, E. (2017). El retorno a la aldea. Neurodiversidad, autismo y electronalidad. Kindle Edition. http://www.librosperuanos.com/libros/detalle/18647/El-retorno-a-la-aldea.-Neurodiversidad-autismo-y-electronalidad

Reaño, E. (2020). Trener contra Reaño. https://ernestoreano.pe/trener-contra-reano/

Reaño, E. (2020). Pensar la conectividad y la electronalidad en tiempos de la COVID-19: el WhatsApp y el modelo comunicativo del autismo. Revista De Investigaciones De La Universidad Le Cordon Bleu, 7(1), 71-77. https://doi.org/10.36955/RIULCB.2020v7n1.005

Shore, S. (2012). A different order of being. En Santomauro J. (Ed.) Autism all-stars. London: Jessica Kingsley Publishers. https://uk.jkp.com/products/autism-allstars

Singer, J. (2016). Neurodiversity. The birth of an idea. Kindle Edition. Judy-Singer-ebook/dp/ B01HY0QTEE

Singer, J. (2020). What is Neurodiversity? https://neurodiversity2.blogspot.com/p/what.html

Warrier, V., Greenberg, D.M., Weir, E., Buckingham, C. Smith, P., Meng-Chuan, L., Carrie, A., y Baron-Cohen, S. (2020). Elevated rates of autism, other neurodevelopmental and psychiatric diagnoses, and autistic traits in transgender and gender-diverse individuals. Nat Commun 11, 3959. https://doi.org/10.1038/s41467-020-17794-1

Walker, N. (2014). What is autism? https://neuroqueer.com/what-is-autism/

World Health Organization (2019). Autism spectrum disorders. https://www.who.int/news-room/fact-sheets/detail/autism-spectrum-disorders 